\title{
Verificação numérica de ábacos e equações para o cálculo da concentração de tensão
}

\author{
Numerical verification of abacuses and equations for the calculation \\ of stress concentration
}

\begin{abstract}
Osmar Alexandre do Amaral Neto

Osmaralexandre22@hotmail.

com

Universidade de Fortaleza

(Unifor)
\end{abstract}

\section{Andressa Lena Mota Palhano \\ andressampalhano@gmail.com Universidade de Fortaleza (Unifor) \\ Ícaro Rodrigues Marques Icarormarques22@hotmail. com \\ Universidade de Fortaleza (Unifor)}

Michel Bruno de Andrade de Sant'ana mbrunosantana@hotmail.com Universidade de Fortaleza (Unifor)

Ítalo Linhares Salomão italosalomao@unifor.com.br Universidade de Fortaleza (Unifor)

\begin{abstract}
Resumo
O referido artigo consiste na verificação das metodologias de cálculo da concentração de tensão em chapas com furo centrado submetidas unicamente ao esforço de tração em uma direção, proposta por Hibbeler, em seu livro de resistência dos materiais, e por Young e Budynas, no livro Roark's Formulas for Stress and Strain. O método de pesquisa adotado consiste no estudo de caso de estrutura comparativa, no qual se utilizou o Método dos Elementos Finitos (MEF) para a modelagem e análise do efeito da concentração de tensões provocado por mudanças bruscas na seção transversal do elemento. Optou-se por utilizar o programa Robot Structural Analysis, da Autodesk, para o desenvolvimento das modelagens, sendo fundamentadas nas condições de contorno e no estado plano de tensão. A partir dos resultados obtidos por meio do estudo comparativo, pode-se verificar, com base nos valores dos erros encontrados, que o ábaco proposto por Hibbeler expressa valores de erros superiores quando comparado com a equação apresentada por Young e Budynas (2002), em que, para o primeiro caso, constata-se um erro máximo de $8,11 \%$ e médio de $5,45 \%$, enquanto no método proposto por Young e Budynas apresenta-se um erro na faixa de 3,14\% e médio de 1,64\%.
\end{abstract}

Palavras-chave: Concentração de tensão. Elementos finitos. Análise comparativa.

\begin{abstract}
The articleconsists in a verification of calculation methods regarding stress concentration in plates with centered hole and submitted only to traction in one direction, proposed by Hibbeler in its book of Mechanics of Materials and by Young and Budynas in the book Roark's Formulas For Stress and Strain. The research method adopted is a case of a comparative structure in which the Finite Element Method (FEM) was used to model and analyze the effect of stress concentration caused by sudden changes in the cross section of the element. It was decided to use Autodesk's Robot Structural Analysis software for develop the models, which are based on the boundary conditions and the plane stress state. Tables are presented with the values of the stress concentration factors obtained with the FEM, which were used in order to perform a comparison with the calculation methods presented by the authors mentioned above. With the results obtained through the comparison, we can point out, based on the values of the errors found, that the abacus proposed by Hibbeler expresses higher error values when compared to the equation presented by Young and Budynas (2002), in which for the first case, There is a maximum error of $8.11 \%$ and average of $5,45 \%$ while in themethod proposed by Young and Budynas there is an error in the range of 3.14\% and average of $1,64 \%$.
\end{abstract}

Keywords: Stress concentration. Finite element. Comparative aalysis.

\section{Introdução}

No exercício da Engenharia, particularmente em áreas que abordam o dimensionamento de elementos que possam suceder à presença de concentradores de tensão, tais como furos para colocação de parafusos, pinos, tubulações elétricas, hidrossanitárias e etc, vistos em projetos de componentes de máquinas ou projetos de elementos estruturais, os profissionais envolvidos devem atribuir uma atenção redobrada a esse efeito, uma vez que as concentrações de tensão podem ser um dos grandes responsáveis por conduzir à falha precoce os elementos estruturais ou mecânicos.

A existência de furos, rasgos, entalhes ou qualquer outra situação em que ocorra uma mudança da seção transversal do elemento, modifica a constância da área e, consequentemente, varia a distribuição de tensão. Nessa situação, a tensão normal máxima no elemento ocorrerá na menor área de seção transversal, manifestando-se, assim, o fenômeno de concentração de tensão, que é descrito e quantificado através do Fator de Concentração de Tensão. 
Osmar Alexandre do Amaral Neto, Andressa Lena Mota Palhano, Ícaro Rodrigues Marques, Michel Bruno de Andrade de Sant'ana, Ítalo Linhares Salomão

Tendo em vista que, na aplicação da engenharia, a distribuição de tensão real não precisa ser determinada, uma vez que o elemento é projetado para resistir apenas à tensão máxima, empregam-se métodos experimentais ou técnicas matemáticas avançadas, que utilizam a teoria da elasticidade, para a determinação dos valores específicos da tensão máxima na seção crítica.

O revés que envolve o efeito da concentração de tensão pode ser melhor visualizado e compreendido através do conceito de linhas de fluxo (Young e Budynas, 2002), como apresentado na figura 1, que representa um elemento em equilíbrio sujeito a um dado carregamento. Assumindo que o carregamento ao qual o elemento está submetido é aplicado em uma dada região e a perturbação das linhas de fluxo se manifesta a uma certa distância, dispondo do furo como meio por onde as linhas de fluxo atuam, tem-se:

Figura 1 - Representação das linhas de fluxo em chapa com elemento concentrador de tensão.

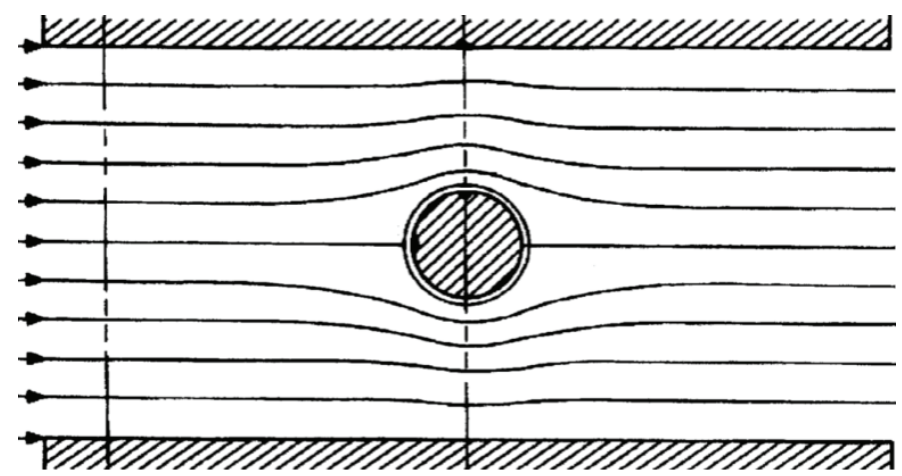

Fonte: Adaptado de Young e Budynas(2002).

Para as seções onde as linhas de fluxo se encontram mais próximas, com base na analogia de linhas de fluxo proposta por Young e Budynas (2002), tem-se que, quanto maior a concentração das linhas de fluxo numa dada região, maior a intensidade destas. Logo, a tensão nesses pontos de concentração de linhas de fluxo torna-se maior quando comparado com as regiões onde as linhas são paralelas, gerando regiões de ocorrência de concentração de tensão.

O problema da concentração de tensão é mais comumente elucidado mediante a aplicação de técnicas numéricas ou experimentais. Dentre os diferentes métodos numéricos para soluções não analíticas, o Método dos Elementos Finitos (MEF), amplamente utilizado em todo o mundo, foi o escolhido como premissa, aliado ao programa Robot Structural Analysis, da Autodesk. O método consiste em subdividir o corpo contínuo da estrutura em um número finito de elementos, constituindo, assim, a malha de elementos finitos, com geometria simples e de fácil solução, obtendo-se o resultando final por meio da superposição da solução de cada elemento.

\section{Fundamentação}

Usualmente, as equações empregadas para os cálculos de resistência dos materiais são formuladas presumindose que os elementos analisados possuem seção transversal constante, ou que estas dimensões sofram modificações de maneira gradual. Porém, situações em que pode ocorrer redução de seção na peça são bastante comuns, o que leva a uma modificação na distribuição de tensão.

Uma interpretação do efeito da concentração de tensão é expressa por Timoshenko (1980), segundo o qual, para análise de tensões em anéis, discos circulares, barras curvas com seção transversal retangular, entre outras, adota-se a utilização de coordenadas polares.

Segundo Timoshenko (1980), ao submeter uma chapa à tração uniforme, sua distribuição de carga também será uniforme, porém, quando se tem uma alteração na seção transversal, ocorre uma perturbação na distribuição da carga ao redor da mesma. O princípio de Saint-Venant assegura que o efeito ocasionado, devido a qualquer mudança de seção ou carregamento aplicado no elemento, dissipa-se ou atenua ao longo do mesmo, em regiões suficientemente afastadas do ponto de aplicação da carga (Hibbeler, 2010). 
Por conseguinte, Timoshenko (1980) analisou uma chapa submetida a uma tração uniforme S com uma delimitação concêntrica e circular de raio b, grande em relação a um orifício de raio a (Figura 2), constatando, pelo princípio supracitado, que as tensões à distância b são as mesmas que para uma chapa sem orifício.

Figura 2 - Distribuição de tensão em chapa com furo centrado.

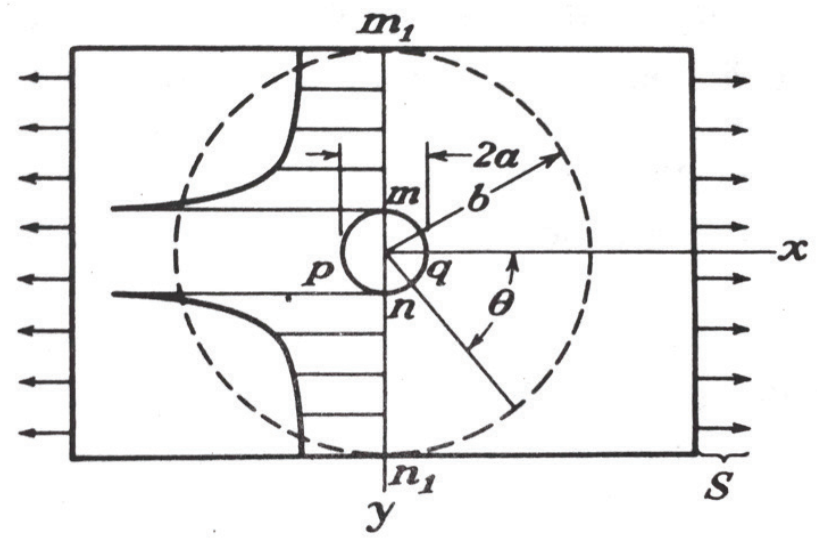

Fonte: Timoshenko (1980).

Por meio de equações diferenciais propostas pela Teoria da Elasticidade e fazendo a consideração da chapa com um furo muito pequeno em relação à altura da mesma, observou-se que a tensão $\sigma_{\theta}$ é máxima quando $\theta=\mathrm{r} / 2$ ou $\theta=3 \mathrm{r} / 2$, i.e., nas extremidades superior e inferior do orifício. O modelo matemático que rege a tensão é dado pela equação abaixo.

$$
\sigma_{\theta}=\frac{S}{2}\left(2+\frac{a^{2}}{r^{2}}+3 \frac{a^{4}}{r^{4}}\right)
$$

Analisando a Eq. 1 para $r$ tendendo ao valor de $\alpha$, sendo $r$ a distância do centro do furo a um ponto qualquer da chapa e $\alpha$ o raio do orifício, tem-se a máxima tensão de tração, sendo ela igual a três vezes a tensão uniforme $[(\sigma)] \theta=3 \mathrm{~S})$.

Define-se o fator K como o quociente entre a tensão máxima e a tensão média que atuam sobre a menor seção transversal do elemento (Eq. 2).

$$
K_{t}=\frac{\sigma_{\text {máx }}}{\sigma_{\text {méd }}}
$$

Young e Budynas (2002) apresenta uma equação para o cálculo do fator de concentração de tensão $\mathrm{K}_{t}$ (Eq. 3) para chapas retangulares com orifício central e submetida a uma carga uniforme, sendo ela:

$$
K_{t}=3,00-3,13\left(\frac{2 r}{D}\right)+3,66\left(\frac{2 r}{D}\right)^{2}-1,53\left(\frac{2 r}{D}\right)^{3}
$$

Outra indagação relacionada à concentração de tensão, segundo Timoshenko (1980), pode ser vista mediante o estudo realizado por David Brewster, em que um pedaço de vidro tensionado, ao ser examinado sob luz polarizada, apresenta uma configuração de cores em escala de cinza devido as tensões (modelo fotoelástico). Dessa forma, o autor sugeriu que, a partir dessas configurações, poderiam ser medidas as tensões para estruturas de engenharia sob diversos carregamentos. Tal premissa só foi aceita anos depois e, posteriormente, melhorados.

Segundo Doyle (2004), o modelo fotoelástico é um método experimental de análise de tensão baseado no fenômeno de birrefringência, encontrado em alguns polímeros quando inseridos sob carga. Por se tratar de um modelo plástico, e não de um protótipo, limitando a variação do comportamento constitutivo, o modelo fotoelástico se restringe a problemas elásticos lineares.

Young e Budynas (2002) estudaram um modelo fotoelástico analisando as tensões encontradas nos pontos a, b e c, apresentado na figura 3. Em seguida, fizeram um comparativo entre os resultados obtidos pela Eq. 3, apresentada por Heywood, e o modelo fotoelástico, no qual obtiveram um erro de $2 \%$. 
Osmar Alexandre do Amaral Neto, Andressa Lena Mota Palhano, Ícaro Rodrigues Marques, Michel Bruno de Andrade de Sant'ana, Ítalo Linhares Salomão

Figura 3 - Modelo Fotoelástico.

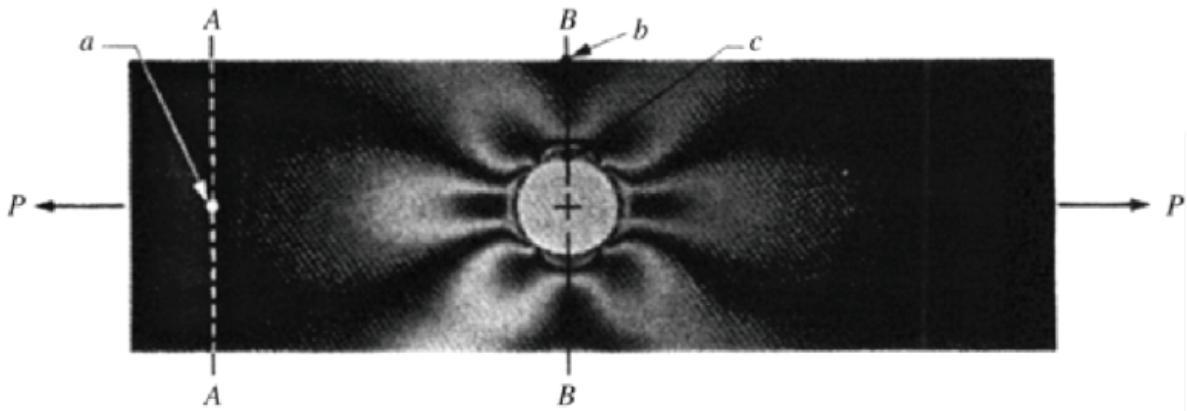

Fonte: Young e Budynas (2002).

Outra abordagem referente à concentração de tensão é apresentada por Hibbeler (2010), em que mostra que, ao se aplicar uma força axial pontual em um elemento, gera-se uma concentração de tensão na região. Outro exemplo apresentado pelo autor ocorre em uma redução de seção ou furo no elemento, mostrando que tende a gerar uma irregularidade na distribuição da tensão, ocasionando tensões máximas na menor área de seção transversal, como apresentado na figura 4.

Levando em consideração que o material possua um comportamento linear elástico, a força resultante, $\mathrm{P}$, devido à distribuição de tensões, pode ser apresentada como visto na Eq. 4.

$$
P=\int_{A} \sigma d A
$$

Figura 4 - Distribuição de tensões em barras com furos e redução de seção.

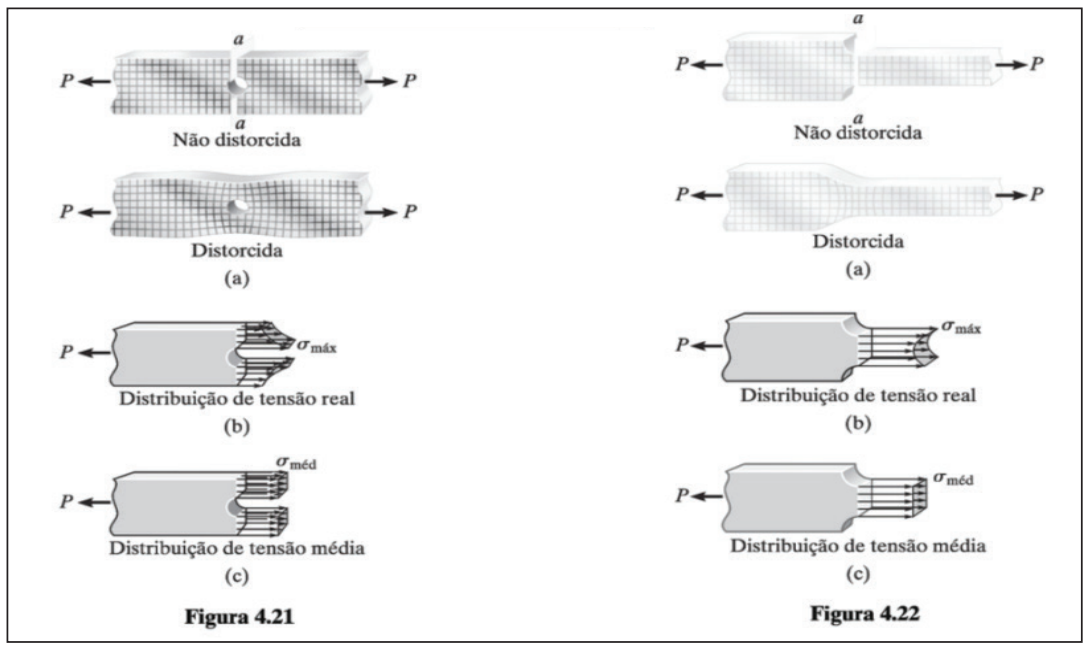

Fonte: Adaptado: Hibbeler (2010).

Ao se projetar um elemento submetido à tração, estima-se que o mesmo deve resistir a uma tensão máxima, $\sigma_{\text {máx }}$, na sua seção, proveniente de um carregamento aplicado axialmente. Quando há a mudança na área transversal do elemento, essa variação pode apresentar valores de $\sigma_{\text {máx }}$, pois são regiões de seção crítica. Os valores podem ser representados graficamente em função do fator de concentração de tensão.

Observa-se que $\mathrm{K}$ depende apenas da geometria do elemento, ou seja, independe das propriedades do material da barra, e podem ser obtidos por gráficos em manuais relacionados à análise de tensão. É apresentado um ábaco na Figura 5, proposto por Hibbeler (2010), o qual relaciona os valores para o fator K que dependem da razão entre o raio e a altura do furo (condição constituinte de descontinuidade na seção). Observa-se que à medida que $\mathrm{r} / \mathrm{w}$ diminui, a concentração de tensão aumenta. 
Na figura 5, seus valores foram determinados com base em um carregamento estático, considerando que as tensões do material mantenham limites de proporcionalidade. Outro ponto importante a ser observado é que esses valores foram determinados experimentalmente.

Figura 5 - Ábaco com valores de K.

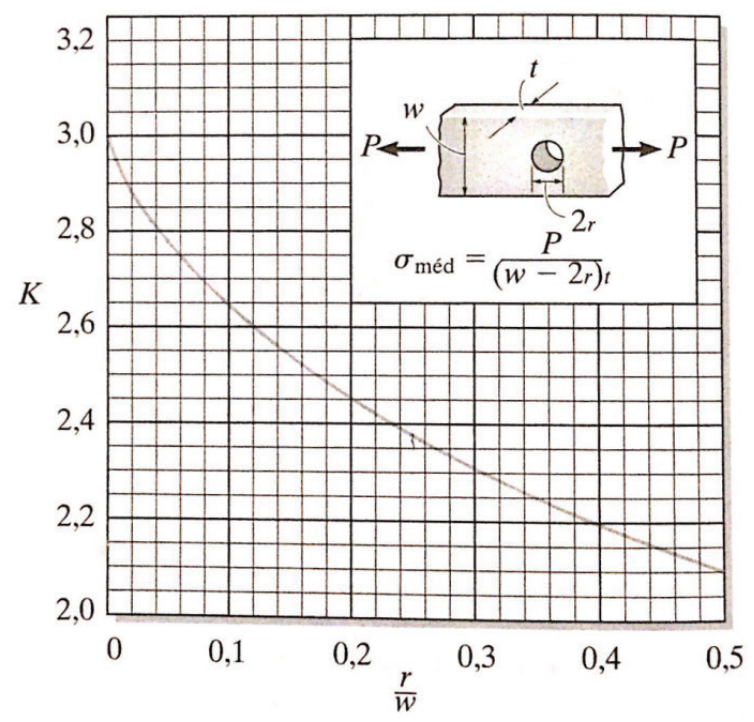

Fonte: Adaptado: Hibbeler(2010).

Como forma de verificar os métodos de cálculo de concentração de tensão expostos anteriormente, foi utilizado o método dos elementos finitos, o qual consiste em uma subdivisão do domínio de uma estrutura em partes menores de dimensões limitadas, chamadas de elementos finitos. Tais subdivisões apresentam diversas formas que, dependendo do tipo de problema e de sua dimensão, podem ser triangulos, quadriláteros, entre outros. Cada elemento deve ser ligado por meio de nós ou pontos nodais, formando uma estrutura denominada "malha".

"O método dos elementos finitos é um método de aproximação por partes, no qual a função de aproximação $\varphi$ é formada conectando funções simples, cada uma definida sobre uma pequena região (elemento). Um elemento finito é uma região no espaço em que uma função $\varphi$ é interpolada a partir de valores nodais de $\varphi$ sobre o limite da região de tal forma que a continuidade entre elementos de $\varphi$ tende a ser mantida no conjunto." (Cook, 1988, tradução do autor).

Segundo Soriano (2003), é aconselhavel que a discretização da malha de elementos finitos obedeça aos seguintes criterios:

1. Em regiões de elevados gradientes de tensão, deve-se diminuir o tamanho do elemento e/ ou aumentar a ordem de interpolação do mesmo.

2. Colocar pontos nodais onde ocorrer apoio elástico, deslocamentos prescritos e forças concentradas, obedecedo a geometria do corpo em estudo. Evitar colocar apoios elásticos em pontos nodais localizados na face do elemento.

3. Utilizar malhas que respeitem a simetria do modelo matemático e, sempre que possivel, utilizar essa simetria na elaboração do modelo discreto.

4. Procurar evitar ao máximo a distorção dos elementos em regiões de elevados gradientes de tensão, como elementos com um lado menor do que três vezes os outros, e angulos internos de vértices menores que $45^{\circ}$ e maiores do que $135^{\circ}$.

5. Evitar o uso de elementos com rigidez muito distintas, sendo mais desejável impor restrições no deslocamento nodal.

De acordo com Cook (1988), a análise de tensões por elementos finitos é composta por sete passos, os quais estão citados nos seguintes itens: 
Osmar Alexandre do Amaral Neto, Andressa Lena Mota Palhano, Ícaro Rodrigues Marques, Michel Bruno de Andrade de Sant'ana, Ítalo Linhares Salomão

1. Dividir a estrutura em elementos finitos com ajuda de programas geradores de malha, chamados pré-processadores.

2. Definir as propriedades de cada elemento, determinando as cargas nodais associadas a todos os estados de deformação que são permitidos ao elemento.

3. Montar o modelo de elementos finitos da estrutura.

4. Aplicar as cargas referentes ao modelo estudado, sejam forças nodais, sejam momentos.

5. Especificar como a estrutura é apoiada, definindo os deslocamentos nodais cujos valores são conhecidos, possuindo muitas vezes valor zero.

6. Resolver equações algébricas lineares para determinar os graus de liberdade nodais.

7. Calcular as deformações de elementos a partir dos graus de liberdade nodais e da interpolação do campo de deslocamento do elemento e, finalmente, calcular as tensões a partir da deformação.

\section{Método}

Nesta pesquisa, foi utilizado, segundo Yin (2010), o método de pesquisa baseado em um estudo de caso de estrutura comparativa, no qual se restringiu o universo do problema para a situação de concentração de tensão em chapas com furos centrados.

O modelo estudado adotou um elemento de forma a diminuir o esforço computacional e atender ao estado plano de tensão, cuja principal característica é possuir somente tensões aplicadas paralelamente à superfície do elemento.

Quanto às condições de carregamento atuantes sobre a estrutura, foi convencionada a aplicação de um esforço externo de tração, correspondente a uma carga uniforme de $1100 \mathrm{KN} / \mathrm{m}$ ao longo de uma das bordas laterais da chapa, estando a outra borda impedida de transladar e rotacionar em qualquer direção, ou seja, em uma situação de engastamento perfeito, conforme pode ser observado na figura 6 .

Figura 6 - Modelo feito com auxílio do programa Robotstructural Analysis.

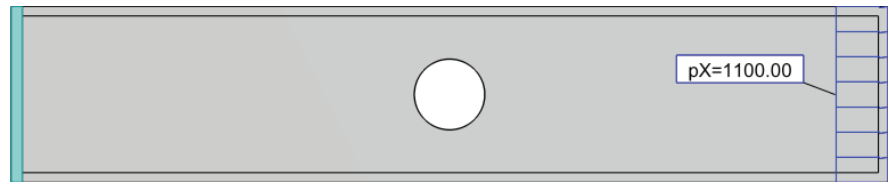

Fonte: Os autores (2017).

Com o intuito de analisar os efeitos das dimensões da chapa na concentração de tensão, foram modelados três tipos de elementos com furo centrado, apresentando 0,5, 1,0 e 1,5 metros de altura, mantendo constante um comprimento de 5 metros e uma espessura de 0,02 metros. É ilustrado na figura 7, as chapas em suas três alturas distintas conforme informação supracitada, com a representação da tensão axial quando esta é submetida a tração. Na figura 7, observa-se que as concentrações de tensão ocorrem exatamente nas bordas superior e inferior do furo, junto a isso, identifica-se a existência de tensões de compressão nas bordas lateral direita e esquerda.

Figura 7 - Concentração de tensão nos modelos de altura $0,5,1,0$ e $1,5 \mathrm{~m}$.

Tensão axial em chapa de $0,5 \mathrm{~m}$ de altura

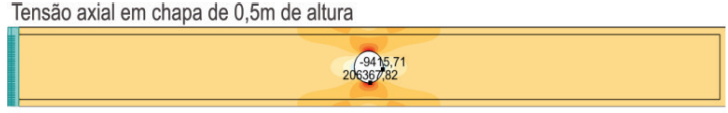

Tensão axial em chapa de 1,0m de altura

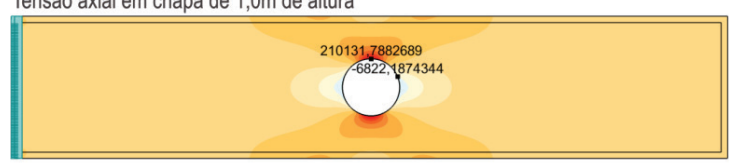

Tensão axial em chapa de $1,5 \mathrm{~m}$ de altura

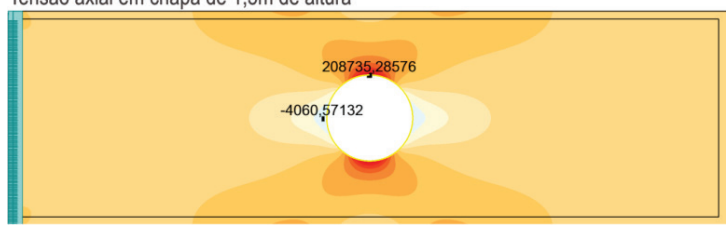

Fonte: Os autores (2017). 
Com o auxílio do programa Robot Structural Analysis e adotando como modelo para concepção do elemento o estado plano de tensão, foram realizadas nove modelagens para cada situação, de acordo com os diferentes diâmetros adotados. $\mathrm{O}$ aço foi o material empregado em todas as modelagens, admitindo suas propriedades constantes, tais como módulo de elasticidade do material $(\mathrm{E}=210 \mathrm{GPa})$ e o coeficiente de Poisson $(v=0,3)$.

$\mathrm{O}$ modelo da malha de elementos finitos fundamentou-se a partir de uma análise visando determinar o tamanho máximo de cada elemento, a validação do modelo adotado, e a convergência dos resultados. Durante o processo de refinamento da malha, à medida que se aumenta o raio do furo, a discretização entre a borda do mesmo e a borda superior da chapa se torna insuficiente, o que levaria a pequenas alterações no formato da peça e consequentes erros. Com o intuito de sanar essa falha ao se discretizar a malha, optou-se por diminuir o tamanho máximo do elemento sem alterar as características geométricas da chapa.

Quanto à forma de geração da malha, optou-se por utilizar a superfície de Coons, a qual consiste em uma superfície paramétrica gerada por meio de mapeamento transfinito, a partir de quatro linhas de fronteira com parametrização genérica, que devem estar ligadas a duas linhas adjacentes por pontos de extremidade.

A discretização adotada pode ser observada na figura 8, que representa a malha de elementos finitos gerada pelo método de coons, cujos parâmetros de configuração são apresentados pelo programa de elementos finitos adotado neste artigo.

Figura 8 - Representação da malha de elementos finitos, gerada utilizando a superfície de coons.

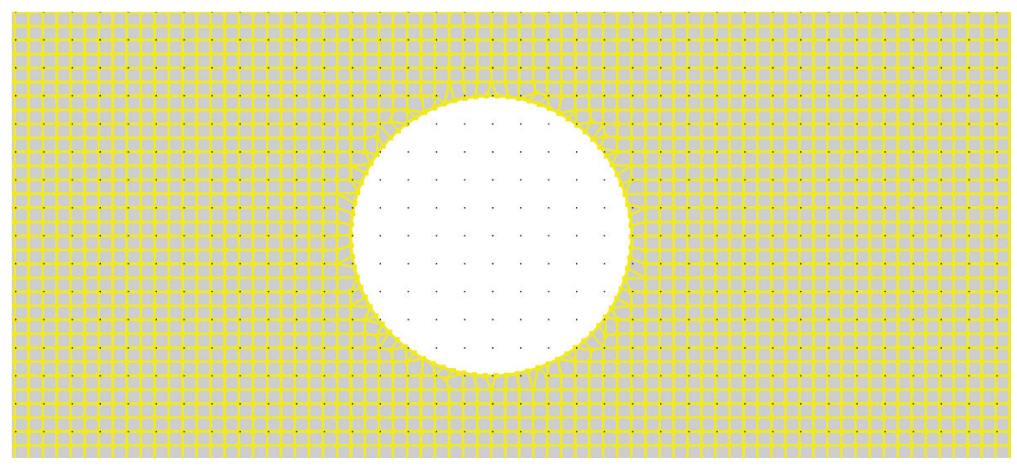

Fonte: Os Autores (2017).

\section{Resultados e discussão}

Com base nos resultados de cada modelagem, tornou-se possível determinar o fator de concentração de tensão Kt, cuja definição consiste na relação entre a tensão máxima e a tensão média, ambas calculadas na região de menor seção da chapa.

Reproduzindo-se o processo de aferição dos dados para cada modelagem, de acordo com os diferentes diâmetros adotados, obtiveram-se nove casos distintos, em que se fez o levantamento dos valores de tensão máxima e fator de concentração de tensão para cada ocorrência, de forma a possibilitar a montagem das tabelas 1, 2 e 3 .

Tais tabelas são compostas da relação entre diâmetro do furo e altura da chapa (D/W), tensão média na menor seção transversal, e a tensão máxima encontrada nas modelagens, as quais, quando relacionadas, resultam no fator Kt. 
Osmar Alexandre do Amaral Neto, Andressa Lena Mota Palhano, Ícaro Rodrigues Marques, Michel Bruno de Andrade de Sant'ana, Ítalo Linhares Salomão

Tabela 1 - Resultados de Kt para chapa de 0,5m de altura utilizando o MEF.

\begin{tabular}{|c|c|c|c|}
\hline $\mathbf{D} / \mathbf{W}$ & TENSÃO MÉDIA & TENSÃO MAXIMA & Kt (MEF) \\
\hline 0,1 & 61111,111 & 164936,6371 & 2,70 \\
\hline 0,2 & 68750,000 & 175325,8311 & 2,55 \\
\hline 0,3 & 78571,429 & 183991,4 & 2,34 \\
\hline 0,4 & 91666,667 & 206367,8186 & 2,25 \\
\hline 0,5 & 110000,000 & 240812,0762 & 2,19 \\
\hline 0,6 & 137500,000 & 298719,7136 & 2,17 \\
\hline 0,7 & 183333,333 & 384180,4623 & 2,10 \\
\hline 0,8 & 275000,000 & 577912,7243 & 2,10 \\
\hline 0,9 & 550000,000 & 1094454,053 & 1,99 \\
\hline
\end{tabular}

Fonte: Os autores (2017).

Tabela 2 - Resultados de Kt para chapa de 1,0m de altura utilizando o MEF.

\begin{tabular}{c|c|c|c}
\hline D/W & TENSÃO MÉDIA & TENSÃO MAXIMA & Kt (MEF) \\
\hline 0,1 & 61111,111 & 169098,234 & 2,77 \\
\hline 0,2 & 68750,000 & 174611,199 & 2,54 \\
\hline 0,3 & 78571,429 & 190031,846 & 2,42 \\
\hline 0,4 & 91666,667 & 210131,788 & 2,29 \\
\hline 0,5 & 110000,000 & 241238,635 & 2,19 \\
\hline 0,6 & 137500,000 & 296322,109 & 2,16 \\
\hline 0,7 & 183333,333 & 387855,928 & 2,12 \\
\hline 0,8 & 275000,000 & 574384,106 & 2,09 \\
\hline 0,9 & 550000,000 & 1127916,197 & \\
\hline
\end{tabular}

Fonte: Os autores(2017).

Tabela 3 - Resultados de Kt para chapa de 1,5m de altura utilizando o MEF.

\begin{tabular}{cccc}
\hline D/W & TENSÃO MÉDIA & TENSÃO MAXIMA & Kt (MEF) \\
\hline 0,1 & 61111,111 & 165839,960 & 2,71 \\
\hline 0,2 & 68750,000 & 177586,300 & 2,58 \\
\hline 0,3 & 78571,429 & 186736,817 & 2,38 \\
\hline 0,4 & 91666,667 & 208735,286 & 2,28 \\
\hline 0,5 & 110000,000 & 240157,500 & 2,18 \\
\hline 0,6 & 137500,000 & 295026,018 & 2,15 \\
\hline 0,7 & 183333,333 & 385663,161 & 2,10 \\
\hline 0,8 & 275000,000 & 573245,495 & 2,08 \\
\hline 0,9 & 550000,000 & 1122630,860 & 2,04 \\
\hline
\end{tabular}

Fonte: Os autores (2017).

Apresentando os dados das tabelas supracitadas, na forma de ábaco (Figura 9), pode-se notar que os resultados não possuem grandes divergências entre si, constatando que as dimensões dos modelos não afetam os resultados de forma significativa. Tal efeito é possível devido à geometria ser suficiente para que as tensões se estabilizem em grandes distâncias do ponto de aplicação de carga. 
Figura 9 - Resultados de Kt utilizando o MEF.

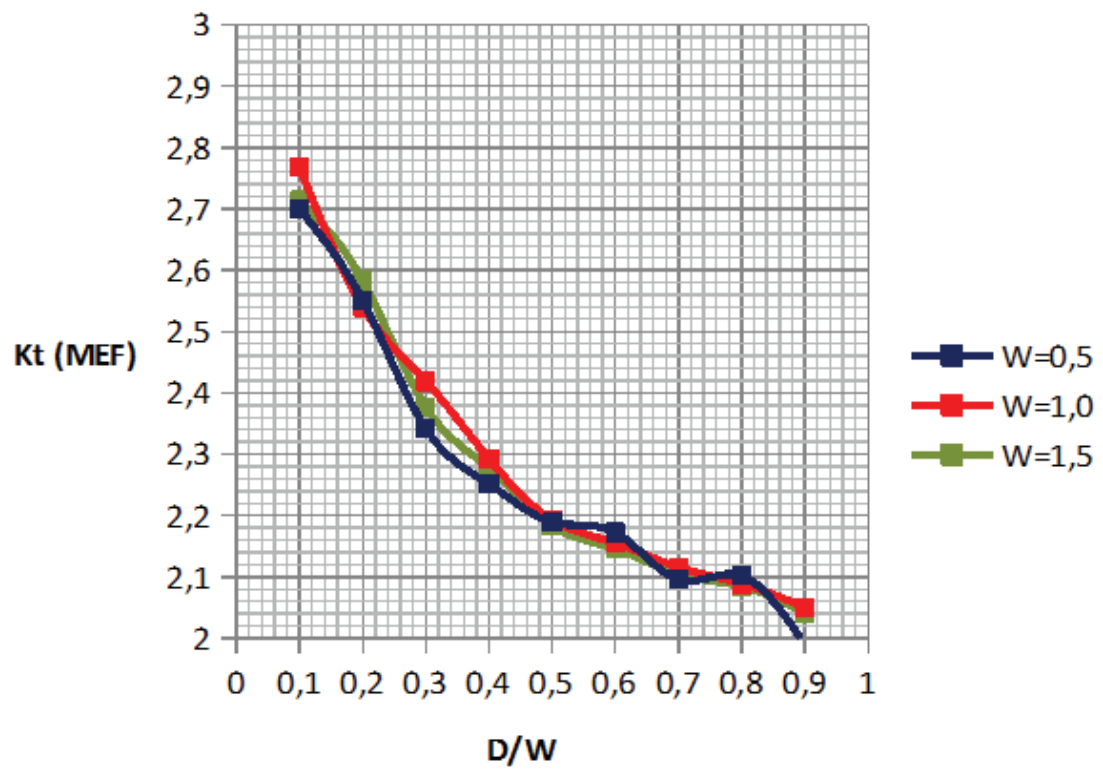

Fonte: Os autores (2017).

Ao ser utilizado, como parâmetro de comparação, o ábaco de concentração de tensão apresentado no livro de resistência dos materiais de Hibbeler (2010) e a equação de Young e Budynas (2002), ambas abordadas anteriormente no referido artigo, percebeu-se que há uma divergência significativa quando comparados os resultados do MEF com o ábaco apresentado por Hibbeler (2010). Em contrapartida, o ábaco confeccionado com base na equação apresentada por Young e Budynas (2002) apresenta resultados mais próximos dos obtidos pelo MEF, sendo essa verificação feita por meio da sobreposição dos resultados gráficos para cada situação (Figura 10).

Figura 10 - comparação dos resultados de Kt com os métodos propostos por Hibbeler e Young e Budynas.

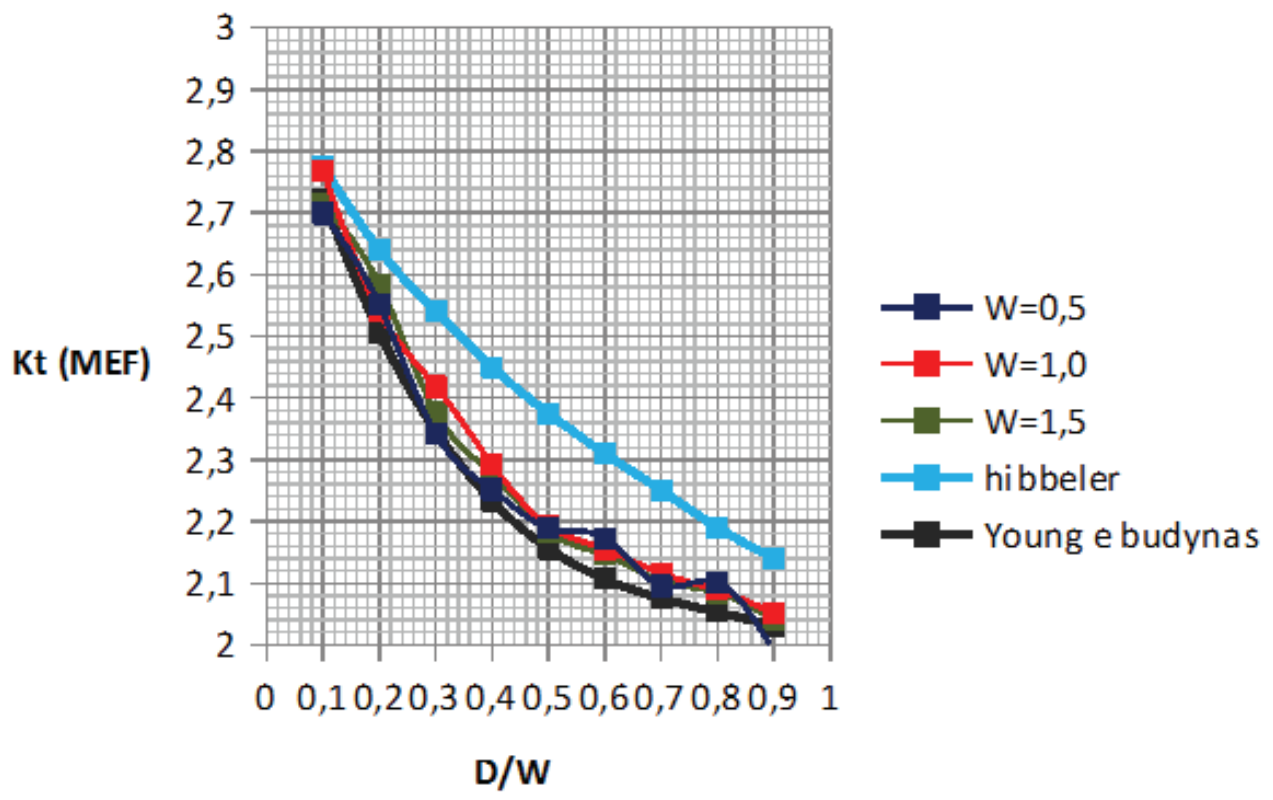

Fonte: Os autores(2017). 
Osmar Alexandre do Amaral Neto, Andressa Lena Mota Palhano, Ícaro Rodrigues Marques, Michel Bruno de Andrade de Sant'ana, Ítalo Linhares Salomão

A Figura 10 evidencia os resultados alcançados, para as diversas chapas em análise e, por meio de superposição destes, faz-se um comparativo com as soluções apresentadas por Hibbeler (2010) e Young e Budynas (2002).

Após a verificação dos resultados obtidos por meio do comparativo entre as 27 (vinte e sete) situações resultantes das diferentes condições que compreendem as modelagens, e com base nos métodos propostos pelos autores mencionados anteriormente, atesta-se que a equação apresentada por Young e Budynas (2002) apresenta maior proximidade dos valores obtidos pelo método dos elementos finitos, enquanto o ábaco proposto por Hibbeler (2010) é mais favorável à segurança, como apresentado na Tab. 4.

Tabela 4 - Tabela de comparação dos resultados de Kt com os métodos propostos por Hibbeler e Young e Budynas.

\begin{tabular}{c|c|c|c|c|c|c}
\hline & \multicolumn{3}{|c|}{ COMPARAÇÃO 1 } & \multicolumn{3}{c}{ COMPARAÇÃO 2 } \\
\hline D/W & $\begin{array}{c}\text { Hibbeler x } \\
\text { MEF(W=0,5) }\end{array}$ & $\begin{array}{c}\text { Hibbeler } \mathbf{M} \\
\text { MEF(W=1,0) }\end{array}$ & $\begin{array}{c}\text { Hibbeler x } \\
\text { MEF(W=1,5) }\end{array}$ & $\begin{array}{c}\text { Young e budynas } \\
\mathbf{x} \text { MEF(W=0,5) }\end{array}$ & $\begin{array}{c}\text { Young e budynas } \mathbf{x} \\
\text { MEF(W=1,0) }\end{array}$ & $\begin{array}{c}\text { Young e budynas } \\
\mathbf{x} \text { MEF(W=1,5) }\end{array}$ \\
\hline 0,1 & $2,74 \%$ & $0,29 \%$ & $2,21 \%$ & $0,82 \%$ & $1,69 \%$ & $0,27 \%$ \\
\hline 0,2 & $3,40 \%$ & $3,80 \%$ & $2,16 \%$ & $1,74 \%$ & $1,33 \%$ & $3,06 \%$ \\
\hline 0,3 & $7,81 \%$ & $4,78 \%$ & $6,43 \%$ & $0,22 \%$ & $3,06 \%$ & $1,27 \%$ \\
\hline 0,4 & $8,11 \%$ & $6,43 \%$ & $7,06 \%$ & $0,82 \%$ & $2,66 \%$ & $1,98 \%$ \\
\hline 0,5 & $7,82 \%$ & $7,66 \%$ & $8,07 \%$ & $1,55 \%$ & $1,73 \%$ & $1,27 \%$ \\
\hline 0,6 & $5,95 \%$ & $6,71 \%$ & $7,11 \%$ & $3,14 \%$ & $2,32 \%$ & $1,87 \%$ \\
\hline 0,7 & $6,87 \%$ & $5,97 \%$ & $6,51 \%$ & $0,99 \%$ & $1,95 \%$ & $1,38 \%$ \\
\hline 0,8 & $4,04 \%$ & $4,63 \%$ & $4,82 \%$ & $2,36 \%$ & $1,73 \%$ & $1,53 \%$ \\
\hline 0,9 & $7,01 \%$ & $4,17 \%$ & $4,62 \%$ & $2,03 \%$ & $0,97 \%$ & $0,50 \%$ \\
\hline
\end{tabular}

Fonte: Os autores(2017).

Analisando os resultados obtidos na tabela supracitada, pode-se apontar, com base nos valores dos erros encontrados, que o ábaco proposto por Hibbeler expressa valor máximo de erro 8,11\% na comparação 1, enquanto que, na comparação 2, referente a Young e Budynas, o erro máximo foi de 3,14\%.

\section{Conclusão}

O artigo teve como método o estudo de caso de estrutura comparativa, na qual foram analisadas diversas literaturas, bem como suas formas de se efetuar o cálculo de concentração de tensão em chapas com furo centrado e submetidas unicamente ao esforço de tração, sendo escolhidas apenas duas literaturas para comparação com os resultados obtidos pelo método dos elementos finitos.

Variadas situações foram obtidas por meio das modelagens, as quais compreendem uma chapa com furo circular de diferentes dimensões e altura variando entre 0,5, 1,0 e 1,5 metros, sendo tal análise necessária como forma de se obter um controle dos efeitos causados pelo princípio de Saint-Venant, de modo que essas implicações não alterem a distribuição de tensão entorno do furo.

Utilizando o método dos elementos finitos por meio do programa Robot Structural Analysis, da Autodesk, foi possível gerar diversos valores para o $\mathrm{K}_{\mathrm{t}}$ e compará-los com os resultados obtidos com o ábaco proposto por Hibbeler (2010) e a equação proposta por Young e Budynas (2002).

Verificou-se que, com relação aos resultados numéricos obtidos, a equação utilizada para a obtenção do $\mathrm{K}_{\mathrm{t}}$, proposta por Young e Budynas (2002) apresenta valores mais próximos aos fornecidos pelo programa, em que se encontra um erro com valor máximo $3,14 \%$ e médio de $1,64 \%$ quando relacionados. Os valores encontrados pelo método empregado por 
Verificação numérica de ábacos e equações para o cálculo da concentração de tensão

Hibbeler (2010) apresentam valores que divergem de modo mais significativo quando comparados aos apresentados pelo programa, com erro de valor máximo $8,11 \%$ e médio de $5,45 \%$.

Young e Budynas (2002), em análises comparativas entre diversos métodos de determinação do fator de concentração de tensões $\mathrm{K}_{\mathrm{t}}$ comentam que um erro de até $5 \%$ é comum para a variação desses resultados. Com base nos resultados obtidos através das análises comparativas pode-se admitir que, de maneira geral, as soluções obtidas foram satisfatórias para as comparações, permitindo uma aferição do panorama que envolve o assunto.

\section{Referências}

COOK, Robert D.; MALKUS, David S.; PLESHA, Michael E. Concepts and applications of finite element analysis. 3.ed. New York: John Wiley \& Sons, 1988.

DOYLE, James F. Modern experimental stress analysis: completing the solution of partially specified problems. Lafayette: John Wiley \& Sons, 2004.

HIBBELER, Russel Charles. Resistência dos materiais. 7. ed. São Paulo: Pearson Prentice Hall, 2010.

SORIANO, Humberto Lima. Método de elementos finitos em análise de estruturas. São Paulo: Edusp, 2003.

TIMOSHENKO, S. P.; GOODIER, J. N. Teoria da elasticidade. 3. ed. Rio de Janeiro: Guanabara Dois, 1980.

YIN, R. K. Estudo de caso: planejamento e métodos. Tradução Ana Thorell. 4. ed. Porto Alegre: Bookman, 2010. YOUNG, W. C.; BUDYNAS, R. G. Roark's formulas for stress and strain.7. ed. Estados Unidos: McGraw-Hill, 2002.

Sobre os autores

\section{Osmar Alexandre do Amaral Neto}

Graduando em Engenharia Civil, Universidade de Fortaleza - Unifor, Coordenador do setor de comunicação da Abece Inovação Fortaleza.

\section{Andressa Lena Mota Palhano}

Engenheira Civil, Universidade de Fortaleza - Unifor, Pós Graduanda em Estruturas de Concreto Armado nas Edificações, Universidade de Fortaleza - Unifor.

\section{Ícaro Rodrigues Marques}

Engenheira Civil, Universidade de Fortaleza - Unifor, Mestrando em Estruturas, Pontifícia Universidade Católica do Rio de Janeiro - PUC

\section{Michel Bruno de Andrade de Sant'ana}

Graduando em Engenharia Civil, Universidade de Fortaleza - Unifor.

\section{Ítalo Linhares Salomão}

Graduado em Engenheiro Civil, Universidade de Fortaleza - Unifor, Mestre em Estruturas, Pontifícia Universidade Católica do Rio de Janeiro - PUC, Professor e Coordenador dos cursos de Engenharia Civil e Construção de Edifícios da Universidade de Fortaleza. 